01

\title{
О модели эффективной среды для частиц со сложной структурой
}

\author{
(С) Л.А. Апресян, Д.В. Власов, Д.А. Задорин, В.И. Красовский
}

Институт общей фризики им. А.М. Прохорова РАН,

119991 Москва, Россия

ฯ e-mail: krasovskii@nsc.gpi.ru

(Поступило в Редакцию 6 апреля 2016 г.)

Рассмотрено обобщение приближения эффективной среды на случай матриц, содержащих макроскопически-неоднородные частицы произвольной структуры (топология кермета). Вид результата существенно зависит от эвристического выбора „эффективных ячеек“, используемых при оценке усредненных по объему значений поля и индукции. Простейший выбор в качестве ячейки частицы в невозмущенном поле приводит к приближению Максвелла-Гарнетта, тогда как самосогласованное приближение эффективной среды отвечает замене невозмущенного поля средним. В качестве примеров описаны случаи частиц с оболочкой, а также статистически анизотропных сред с одним выделенным направлением.

DOI: 10.21883/JTF.2017.01.44011.1841

\section{Введение}

Приближение эффективной среды (в англоязычной литературе Effective Medium Approximation, EMA), аналогичное приближению когерентного потенциала в теории твердого тела $[1,2]$, является одним из базовых при описании эффективных параметров сильно неоднородных композитных сред. Предложенное впервые Браггеманом [3] для сред с макроскопическими включениями оно нашло широкие применения в качестве распространенной аппроксимации и при описании композитов, содержащих наноструктурные объекты, например, графены и углеродные трубки [4-6], а также метаматериалов, т.е. сложных композитных сред с отрицательными значениями эффективной диэлектрической и/или магнитной проницаемостей [7,8]. Давая в целом верное качественное описание задачи, это приближение послужило основой для создания целого ряда разнообразных модификаций [9-11], позволяющих перейти к правильному количественному описанию конкретных экспериментов.

Основным методическим преимуществом приближения Браггемана, помимо возможности выхода за пределы применимости обычной теории возмущений, является прежде всего получение качественного описания возникновения порога протекания на основе предельно упрощенных модельных соображений о структуре среды. Кроме того, вдали от порога протекания для эффективных параметров среды ЕМА дает результаты, которые могут не слишком сильно отличаться от результатов другого широко используемого эвристического приближения - модели Максвелла-Гарнетта (Maxwell-Garnett Approximation, MGA) [12]. Последнее хотя и не дает порога протекания, но зато описывает так называемую оптическую аномалию, представляющую собой коллективный резонанс оптической проводимости в многочастичной среде $[13,14]$.

Известно, что эффективные параметры случайнонеоднородных композитных сред существенно зависят не только от объемных соотношений, но и от топологии, т.е. структурного распределения составляющих среду компонент $[15,16]$. В общем случае объемные компоненты позволяют определить лишь границы эффективных параметров, которые можно сузить, если известна какая-либо дополнительная информация о структуре среды [17]. Поэтому предложенное в литературе множество формул для определения эффективных параметров лишь по соотношению составляющих среду компонент, не учитывающих особенностей пространственного распределения (см., например, [18,19] и цитированную там литературу), фактически охватывает целый класс сред с различными микроструктурами, причем из этого множества нельзя выбрать какое-либо одно „наилучшее“ приближение, пригодное на все случаи жизни. При использовании более сложных моделей „оптимальность“ того или иного приближения должна определяться разумным компромиссом между сложностью модели (и соответственно количеством имеющихся в ней свободных параметров) и точностью описания экспериментов.

Обычно EMA, как и MGA, используются для модели статистически изотропной среды, в которой в среднем отсутствуют выделенные направления, так что все статистические характеристики среды инвариантны относительно вращений. Значительно более сложный случай статистически анизотропных сред стал привлекать повышенное внимание лишь относительно недавно. В работе Страуда [20] (см. также обзор [21]) был дан элегантный вывод наиболее общей формы ЕMА, охватывающий случай квазистатического приближения для многокомпонентных анизотропных сред с поликристаллическими включениями. При этом вместо скалярных эффективных параметров, отвечающих изотропному случаю, возникают тензорные величины.

Изложенная в [20] методика, справедливая в общем случае анизотропных сред, ориентирована прежде всего на случай среды с однородными вкраплениями эллиптической формы. Такая аппроксимация применима ко многим практическим задачам, однако она не позволяет 
описать специфические эффекты, связанных с неоднородностью частиц и заметным отличием их формы от эллиптической. Если в случае MGA учет таких эффектов достаточно прост, так что вычисление эффективной диэлектрической проницаемости сводится к определению средней поляризуемости частицы (см. [22], где рассмотрены случаи разнообразных неоднородных частиц), то приближение ЕМА для неоднородных частиц представляется не вполне тривиальным, и насколько нам известно, до сих пор не рассматривалось в литературе.

В настоящей работе хорошо известная простая схема вывода MGA [22] распространяется на общий случай EMA для анизотропной случайной среды с неоднородными частицами. При этом мы ограничиваемся квазистатическим приближением, основным условием применимости которого является малость размеров частиц по сравнению с характерной длиной волны.

\section{1. Общая схема получения приближенных уравнений для эффективных параметров дискретных макроскопически- неоднородных сред}

Рассмотрим случайно-неоднородную среду, состоящую из однородной матрицы с помещенными в нее рассеивающими, в общем случае неоднородными частицами (рис. 1). Материалу матрицы припишем индекс 1, а материалу частиц - индекс 2 , так что точки $r_{1}$ относятся к матрице, а $r_{2}$ к материалу частиц. Это позволяет чисто формально говорить о двухкомпонентной среде с объемом матрицы $V_{1}$ и частиц $V_{2}\left(V=V_{1}+V_{2}-\right.$ полный объем среды). При этом рассматриваемая система полностью описывается распределением диэлектрической проницаемости $\varepsilon(r)$, причем $\varepsilon_{1}=\varepsilon\left(r_{1}\right)=\mathrm{const}$, a $\varepsilon\left(r_{2}\right)$ меняется внутри каждой частицы по некоторому известному закону (в частном случае однородных частиц $\varepsilon\left(r_{2}\right)=\varepsilon_{2}=$ const $)$.

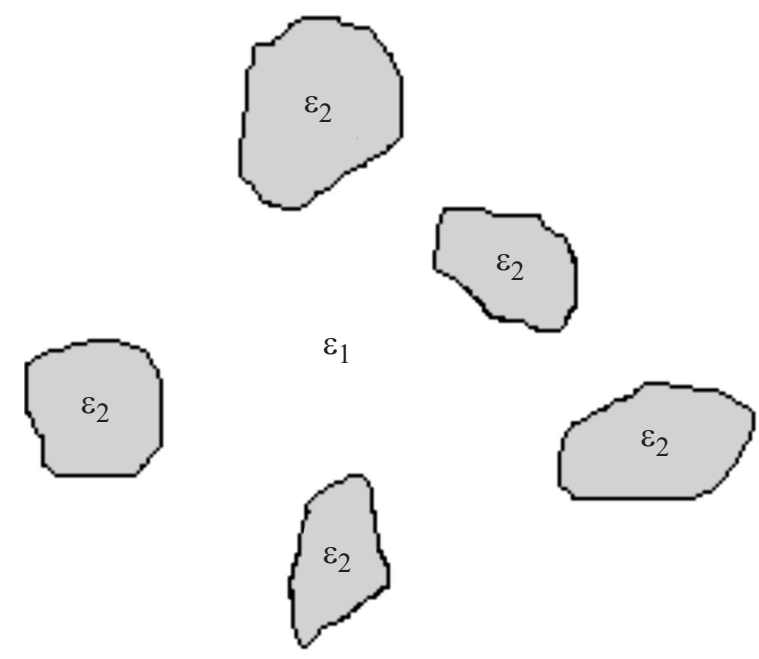

Рис. 1. Модель среды с дискретными вкраплениями.
Будем считать, что такая среда помещена в однородное внешнее поле $E_{1}$, создаваемое удаленными источниками. В результате возникает некоторое сложное распределение поля $E(r)$, отвечающее наличию в среде рассеивающих частиц, причем локальное значение индукции в каждой точке выражается как $D(r)=\varepsilon(r) E(r)$.

Эффективная диэлектрическая проницаемость среды $\varepsilon^{*}$ в квазистатическом приближении по определению описывается соотношением

$$
\langle D\rangle=\varepsilon^{*}\langle E\rangle
$$

где угловые скобки означают пространственное усреднение

$$
\langle F\rangle=\frac{1}{V} \int_{V} F(r) d r
$$

(здесь $F=F(r)$ - произвольная функция $r$; отметим, что в общем случае неоднородного внешнего поля $\varepsilon^{*}$ является оператором, учитывающим пространственную дисперсию).

Из (1) имеем

$$
\begin{aligned}
\varepsilon^{*} & =\langle D\rangle /\langle E\rangle \\
& =\left(\int_{V_{1}} \varepsilon E d r_{1}+\int_{V_{2}} \varepsilon E d r_{2}\right) /\left(\int_{V_{1}} E d r_{1}+\int_{V_{2}} E d r_{2}\right)
\end{aligned}
$$

(обоснование этой упрощенной записи, широко используемой в литературе [23], дано в Приложении 1).

Выражение (3) легко преобразуется к виду

$$
\varepsilon^{*}=\left(f_{1}\langle\varepsilon E\rangle_{V_{1}}+f_{2}\langle\varepsilon E\rangle_{V_{2}}\right) /\left(f_{1}\langle E\rangle_{V_{1}}+f_{2}\langle E\rangle_{V_{2}}\right),
$$

где $f_{j}=V_{j} / V$ - объемная доля, а

$$
\langle F\rangle_{V_{j}}=\frac{1}{V_{j}} \int_{V_{j}} F(r) d r
$$

- усреднение по объему $j$-й компоненты. Здесь $j=1$ для точек матрицы и $j=2$ для точек внутри частиц, причем $f_{1}+f_{2}=1$; если, в частности, усредняемая величина $F$ постоянна внутри $V_{j}$, то $\langle F\rangle_{V_{j}}=F$, что объясняет удобство использования символов усреднения по парциальным объемам.

Подчеркнем, что выражения (3), (4) в рамках квазистатического приближения являются точными и могут использоваться для вычисления $\varepsilon^{*}$, если только считать известным распределение поля в среде $E(r)$. Это поле можно рассчитать численно, если задать конкретную структуру распределения частиц в среде, хотя для реальных случайных сред точный расчет $E(r)$ представляется неосуществимым. Однако, поскольку $E(r)$ входит в (4) в виде усреднения по объему каждой из компонент, которое сглаживает флуктуации поля, при вычислении $\varepsilon^{*}$ можно использовать некоторые достаточно обоснованные эвристические оценки $E(r)$, что, 
собственно, и является основой успеха приближений типа EMA или MGA.

Для реальных рассеивающих сред характеристики частиц являются случайными величинами. При этом аддитивные величины обладают свойством самоусреднения, т.е. их флуктуации затухают с ростом числа составляющих их компонент, что позволяет отождествлять средние арифметические со статистическим усреднением [24]. Так, полный объем второй фазы (частиц) $V_{2}$ есть сумма случайных объемов частиц $v_{k}$ :

$$
V_{2}=\sum_{k=1}^{N} v_{k}=N_{v}
$$

где $v=\frac{1}{N} \sum_{k=1}^{N} v_{k}-$ средний объем частицы, флуктуациями которого при больших $N$ можно пренебречь (для простоты мы отвлекаемся здесь от флуктуаций числа частиц $N$ в рассматриваемой системе, считая $N \gg 1$ ).

Примем естественное допущение, что все частицы статистически эквивалентны в том смысле, что они дают одинаковый вклад в усредненные величины. Тогда усреднение по объему $V_{2}$, содержащее среднее арифметическое по частицам, можно заменить статистическим усреднением, сведя последнее к усреднению по объему $v$ одиночной „эффективной“ частицы:

$$
\langle F\rangle_{V_{2}}=\frac{1}{N v} \sum_{k=1}^{N} \int \theta_{k}(r) F d r=\frac{1}{v} \int_{v} F^{*} d r \equiv\left\langle F^{*}\right\rangle_{v}
$$

Здесь $\theta_{k}(r)$ - характеристическая функция $k$-й частицы, равная единице в объеме частицы и нулю вне его, а

$$
F^{*}=\frac{1}{N} \sum_{k=1}^{N} \theta_{k}(r) F_{k} \equiv\langle F\rangle_{N}
$$

- результат усреднения $F$ по $N$ частицам.

В рассматриваемых далее приближениях величина $F^{*}$ заменяется приближенным значением, которое отвечает используемой модели „средней“ частицы и описывает наиболее характерный „эффективный“ акт рассеяния или иначе „эффективную рассеивающую ячейку“. В результате усреднение по всем внутренним точкам ансамбля частиц $\langle\ldots\rangle_{V_{2}}$ (т.е. „По фазе частиц“) сводится к усреднению по объему „эффективного“ рассеивателя $v$, которое, очевидно, должно быть дополнено статистическим усреднением по всем другим случайным параметрам модели, описывающим ориентацию, состав, форму и другие характеристики случайных рассеивающих частиц.

Соотношение (4) позволяет яснее понять физический смысл $j$-й „эффективной рассеивающей ячейки“, которая используется для оценки поля в точках $j$-й среды. Действительно, в рассматриваемой схеме „эффективные ячейки“ вводятся для оценки статистических характеристик системы „с точки зрения“ внутренних точек среды $\left(r_{1}\right)$ или частиц $\left(r_{2}\right)$, причем среде и частицам, очевидно, должны отвечать в общем случае разные ячейки, поскольку распределение поля внутри и вне частиц существенно различно (исключая относительно простой случай оптически мягких частиц). Выбор таких ячеек неоднозначен и основывается на дополнительных эвристических соображениях. При этом разным выборам $F^{*}$ будут отвечать разные приближения для $\varepsilon^{*}$, которые, вообще говоря, могут описывать среды с разной микроструктурой.

Рассматриваемая здесь задача аналогична классической задаче о переходе от микроописания, когда рассеивающими элементами являются отдельные молекулы, к макроописанию на языке распределения диэлектрической проницаемости. В последнем случае, однако, возникает отдельный вопрос о выборе формы „эффективной рассеивающей ячейки“, которую обычно связывают с наличием сингулярности у функций Грина для уравнений Максвелла [25]. Для выделения этой особенности обычно используют бесконечно малую сферу, что вполне оправдано в случае изотропных сред, но становится не очевидным при переходе к анизотропному случаю. В отличие от этого при использованной в настоящей работе макроскопической постановке задачи „эффективные ячейки“, связанные с частицами, естественно привязываются к усреднению по характеристикам одиночных частиц. Однако для точек среды выбор „эффективной ячейки“ остается целиком основанным на использовании наводящих эвристических соображений.

\section{2. Приближение Максвелла-Гарнетта}

Проиллюстрируем сказанное сначала на относительно простом примере MGA. При получении $\varepsilon^{*}$ в этом приближении поле вне частиц принимается равным полю, создаваемому удаленными источниками в отсутствие частиц, так что $E\left(r_{1}\right)=E_{1}$, а каждая частица заменяется однородной сферой равного с ней объема (рис. 2,a). В случае сферических частиц „огрубление“ задачи в этом приближении связано, очевидно, с отбрасыванием во внешнем по отношению к каждой частице поле вклада от рассеяния на соседних частицах, т.е. замене истинного поля между частицами на однородное поле $E_{1}$, такое же, как и в отсутствие частиц. Отметим, что такая замена не означает полного пренебрежения многократным рассеянием, поскольку полученное при

$a$

$b$

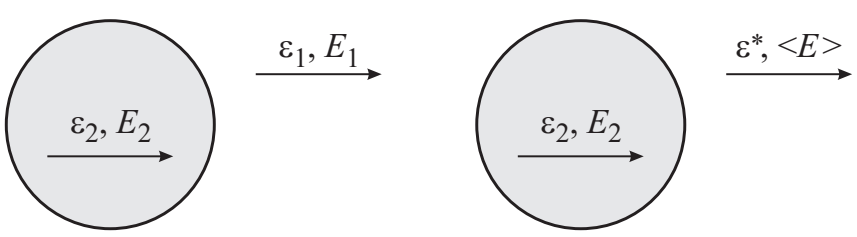

Рис. 2. Модели эффективных рассеивателей в приближениях MGA $(a)$ и EMA $(b)$. 
этом $\varepsilon^{*}$ может существенно отличаться от проницаемости среды $\varepsilon_{1}$ и в конечном итоге рассматривается как локальное значение эффективной диэлектрической проницаемости в усредненных уравнениях Максвелла, последующее решение которых позволяет эффективно учесть многократное рассеяние.

Поле внутри сферической частицы в однородном внешнем поле $E_{1}$ является однородным и выражается известным соотношением [22]

$$
E_{2} \equiv E\left(r_{2}\right)=\Lambda E_{1},
$$

где

$$
\Lambda=\frac{3 \varepsilon_{1}}{\varepsilon_{2}+2 \varepsilon_{1}} .
$$

Поскольку все поля, входящие в (3) под знаками усреднений по $V_{1}$ и $V_{2}$ в этом случае однородны, используя (5) и (6) в (3), получаем

$$
\varepsilon^{*}=\left(f_{1} \varepsilon_{1} E_{1}+f_{2} \varepsilon_{2} \Lambda E_{1}\right) /\left(f_{1} E_{1}+f_{2} \Lambda E_{1}\right)
$$

или после сокращения на $E_{1}$

$$
\varepsilon^{*}=\left(f_{1} \varepsilon_{1}+f_{2} \varepsilon_{2} \varepsilon \Lambda\right) /\left(f_{1}+f_{2} \Lambda\right) .
$$

Подставляя сюда $\Lambda$ (9), получаем обычное выражение для $\varepsilon^{*}$ в приближении MGA, которое может быть записано также в виде известного уравнения [22]

$$
\frac{\varepsilon^{*}-\varepsilon_{1}}{\varepsilon^{*}+2 \varepsilon_{1}}=f_{2} \frac{\varepsilon_{2}-\varepsilon_{1}}{\varepsilon_{2}+2 \varepsilon_{1}}
$$

или в форме уравнения Клаузиуса-Моссотти

$$
\frac{\varepsilon^{*}-\varepsilon_{1}}{\varepsilon^{*}+2 \varepsilon_{1}}=\frac{n \alpha}{3 \varepsilon_{1}}
$$

где

$$
\alpha=v\left(\varepsilon_{2}-\varepsilon_{1}\right) \Lambda
$$

- поляризуемость сферической частицы, а $n=f / v$ плотность числа частиц.

Соотношение (12) часто используется для обобщения MGA на случай других моделей рассеивающих частиц путем простой замены в правой части (12) поляризуемости сферической частицы $\alpha$ на аналогичную поляризуемость более сложных частиц. Однако рассматриваемая здесь схема вывода MGA в случае более сложных моделей приводит к несколько другим результатам, не позволяя выразить $\varepsilon^{*}$ через одну только поляризуемость. Так, например, если вместо однородных сфер вполне аналогично (11) рассмотреть модель одинаково ориентированных однородных эллипсоидов, то $\varepsilon^{*}$ станет зависеть уже не только от поляризуемости, но и от фактора деполяризации эллипсоида $L$, а (12) перейдет в соотношение

$$
\frac{\varepsilon^{*}-\varepsilon_{1}}{\varepsilon^{*}+2 \varepsilon_{1}}=\frac{n \alpha}{3 \varepsilon_{1}}\left(1+\frac{n \alpha}{3 \varepsilon_{1}}(1-3 L)\right)^{-1},
$$

где $\alpha-$ поляризуемость, а $L-$ фактор деполяризации эллипсоида (при этом тензорный характер входящих в (14) величин не приводит к формальным усложнениям, см. ниже разд. 5). В отличие от уравнения Краузиуса-Моссотти (11) здесь правая часть зависит уже не только от $\alpha$, но и от фактора деполяризации эллипсоида $L$ (в случае сфер $L=1 / 3$ и (14), естественно, сводится к (12)).

Существенно, что соотношение (3) при сделанных предположениях может быть легко использовано и для более общих моделей рассеивателей, в частности для неоднородных частиц. В последнем случае поле внутри частицы, находящейся в однородном внешнем поле $E_{1}$, в общем случае будет уже неоднородным, и выражение (8) принимает вид

$$
E\left(r_{2}\right)=\Lambda\left(r_{2}\right) E_{1},
$$

где $\Lambda\left(r_{2}\right)$ - тензор. При этом входящие в (4) операции усреднения по объему частиц (4) становятся нетривиальными, т.е. требуют выполнения интегрирования, и вместо (10) нетрудно получить более общее соотношение

$$
\varepsilon^{*}=\left(f_{1} \varepsilon_{1}+f_{2}\langle\varepsilon(r) \Lambda(r)\rangle_{v}\right) /\left(f_{1}+f_{2}\langle\Lambda(r)\rangle_{v}\right) .
$$

Входящие сюда операции усреднения по объему частицы зависят от принятой модели распределения $\varepsilon(r)$ внутри частицы, которое, в свою очередь, определяет решение задачи о поле внутри частицы, т.е. величину $\Lambda(r)$ из (16). Соотношения такого вида использовались в [22] для оценки влияния различных моделей частиц (в том числе эллиптических, слоистых или более сложно неоднородных) на эффективные параметры среды в MGA.

\section{3. Модель эффективной среды (ЕМА)}

Как отмечалось выше, приближение MGA не учитывает взаимное влияние частиц. ЕМА представляет альтернативный широко распространенный подход к определению эффективных параметров, в котором это влияние учитывается самосогласованно. Получим сначала приближение ЕМА в рамках рассматриваемой здесь схемы для простейшей и наиболее распространенной модели сферических частиц, после чего рассмотрим обобщение на более сложные модели.

Будем считать, как обычно при получении ЕMA, что „эффективный акт рассеяния“ для каждой из компонент $j=1,2$ отвечает модельной частице, находящейся в „эффективной“ среде с проницаемостью $\varepsilon^{*}$. Тогда внешним по отношению к частице выступает уже не поле источников $E_{1}$, как это было в EMG, а среднее поле $\langle E\rangle$. Принимая простейшую модель однородного сферического „эффективного“ рассеивателя, можно использовать соотношения вида (5) и (6), так что

$$
E\left(r_{j}\right)=\Lambda_{j}\langle E\rangle,
$$

где

$$
\Lambda_{j}=\frac{3 \varepsilon^{*}}{\varepsilon_{j}+2 \varepsilon^{*}}
$$


В результате вместо (4) получаем

$$
\varepsilon^{*}=\left(f_{1} \varepsilon_{1} \Lambda_{1}\langle E\rangle+f_{2} \varepsilon_{2}\langle E\rangle\right) /\langle E\rangle=f_{1} \varepsilon_{1} \Lambda_{1}+f_{2} \varepsilon_{2} \Lambda_{2},
$$

или, домножив $\varepsilon^{*}$ на $f_{1}+f_{2}=1$ и перенеся вправо,

$$
0=f_{1}\left(\varepsilon_{1} \Lambda_{1}-\varepsilon^{*}\right)+f_{2}\left(\varepsilon_{2} \Lambda_{1}-\varepsilon^{*}\right) .
$$

После подстановки сюда (18) и простых преобразований получаем обычное уравнение ЕМА для $\varepsilon^{*}$ :

$$
0=f_{1} \frac{\varepsilon_{1}-\varepsilon^{*}}{\varepsilon_{1}+2 \varepsilon^{*}}+f_{2} \frac{\varepsilon_{2}-\varepsilon^{*}}{\varepsilon_{1}+2 \varepsilon^{*}} .
$$

Если для сферических частиц или в более общем случае хаотически ориентированных частиц произвольной формы модель сферического „эффективного рассеивателя“ представляется вполне естественной, то для матрицы, точки которой $r_{1}$ образуют односвязную область, далекую по форме от сферы, использование приближения (14) выглядит менее обоснованным. Тем не менее приближение (21) удовлетворяет правильным „граничным условиям“ $\varepsilon^{*}=\varepsilon_{j}$ в предельных случаях однородного заполнения однородного $f_{j}=1$, а также предположению о статистической изотропности среды.

При этом уравнение (21) симметрично относительно компонент среды, т.е. не меняется при замене местами индексов 1 и 2. Такая симметрия вполне оправдана при описании бесструктурных смесей, однако в рассматриваемом здесь случае с выделенной матрицей 1 представляется искусственным „вырождением“", связанным с использованием одинаковых „эффективных ячеек“ для частиц и среды. В случае более сложных, отличных от однородных сфер рассеивателей эта симметрия пропадает. При этом в случае неоднородных частиц граничное условие $f_{2}=1$ отвечает уже неоднородной среде и, как следствие, не допускает упрощения задачи.

Рассматриваемая схема позволяет легко обобщить уравнение ЕMA (21) на случай более сложных, чем простые сферы, рассеивателей, в том числе различных моделей неоднородных частиц. Для этого в полной аналогии с рассмотренным выше приближением MGA (12) достаточно задать в качестве „эффективного рассеивателя $2^{\text {“ }}$ какую-либо неоднородную структуру, наиболее близко отвечающую рассматриваемому конкретному случаю. При этом решение задачи о поляризации такой структуры в однородном внешнем поле считается известным, т. е. задан явный вид $\Lambda\left(r_{2}\right)$ в $(11)$. В случае статистической изотропности для материала среды (т.е. точек $r_{1}$ ) в качестве ,эффективного рассеивателя“ естественно использовать простейшую модель однородной сферической ячейки (в анизотропном случае „оптимальность“ такого выбора ячейки для точек $r_{1}$ неочевидна, и можно рассматривать в качестве „ячеек среды“, например, эллипсоиды). При этом из (4) вместо (19) получаем

$$
\begin{aligned}
\varepsilon^{*} & =\left(f_{1}\langle\varepsilon E\rangle_{V_{1}}+f_{2}\langle\varepsilon E\rangle_{V_{2}}\right) \\
& =f_{1} \varepsilon_{1} \frac{3 \varepsilon^{*}}{\varepsilon_{1}+2 \varepsilon^{*}}+f_{2}\left\langle\varepsilon\left(r_{2}\right) \Lambda\left(r_{2}\right)\right\rangle_{V_{2}} .
\end{aligned}
$$

Здесь первое слагаемое в правой части отвечает точкам среды, отклик которых на внешнее поле моделируется однородной сферической ячейкой, а второе зависит неявно от $\varepsilon^{*}$. Для получения этой зависимости нужно задать вид ,эффективного рассеивателя““, т. е. $\Lambda(r)$, причем при переходе от усреднения по $V_{2}$ в соответствии с (6) и (7) к эффективной ячейке усреднение по объему $v$ дополняется усреднением по случайным параметрам модели.

Так, например, в модели полностью хаотически ориентированных однородных эллипсоидов тензор $\Lambda(r)$ имеет вид (27) и не зависит от $r$, а $\varepsilon^{*}$ остается скаляром. При этом усреднение по ориентациям в (22), как легко показать, сводится к замене символа усреднения на одну треть от следа, так что (22) переходит в

$$
\begin{aligned}
\varepsilon^{*}= & f_{1} \varepsilon_{1} \frac{3 \varepsilon^{*}}{\varepsilon_{1}+2 \varepsilon^{*}}+f_{2} \varepsilon_{2} \frac{1}{3} \operatorname{Sp} \Lambda=f_{1} \varepsilon_{1} \frac{3 \varepsilon^{*}}{\varepsilon_{1}+2 \varepsilon^{*}} \\
& +f_{2} \varepsilon_{2} \frac{1}{3} \sum_{j=1}^{3} \varepsilon^{*}\left(\varepsilon^{*} 1+L_{j}\left(\varepsilon_{2}-\varepsilon^{*}\right)\right)^{-1}
\end{aligned}
$$

(здесь $L-$ фактор деполяризации эллипсоида, см. (27)). Если в модели сферических частиц EMA (21) сводится к алгебраическому уравнению второго порядка относительно $\varepsilon^{*}$, то (23) приводится уже к уравнению четвертого порядка.

Если использовать (23) для оценки порога протекания $f_{2 c}$, заменив диэлектрическую проницаемость на проводимость, то, как легко показать, из (23) следует выражение

$$
f_{2 c}=\left(\frac{1}{3} \operatorname{Sp} \frac{1}{L}\right)^{-1}=3 / \sum_{j=1}^{3} \frac{1}{L_{j}} .
$$

Такое же выражение для порога протекания получается и в более сложных моделях с эллипсоидальными частицами (если, например, в качестве „ячейки среды“ взять хаотически ориентированный однородный эллипсоид), в этом смысле выражение (24) устойчиво относительно выбора „ячейки среды“.

Это выражение принимает максимальное значение $f_{2 c}=1 / 3$ при переходе от эллипсоида к сфере $\left(L_{1}=L_{2}=L_{3}=1 / 3\right)$. В случае эллипсоида вращения $\left(L_{1}=L_{2} \neq L_{3}\right)$, когда для полуосей эллипсоида $a_{j}$ справедливо соотношение $a_{1}=a_{2} \neq a_{3}$, нетрудно выразить факторы деполяризации эллипсоида через отношение $A=a_{3} / a_{1}$, используя хорошо известные выражения для $L_{j}$ [26]. Результаты такого расчета показаны на рис. 3, откуда видно, что наибольшее значение порога протекания в такой модели достигается в случае сферических рассеивателей, причем любое отклонение от сферичности приводит к уменьшению порога протекания, который обращается в нуль как для предельно вытянутых эллипсоидов („нанотрубки“, $A \rightarrow \infty)$, так и для сплюснутых эллипсоидов („графены“, $A \rightarrow 0$ ). Если, как это делается обычно, определить аспектное отношение $A^{\prime}$ как отношение наибольшего размера частицы к наименьшему, то 
$a$
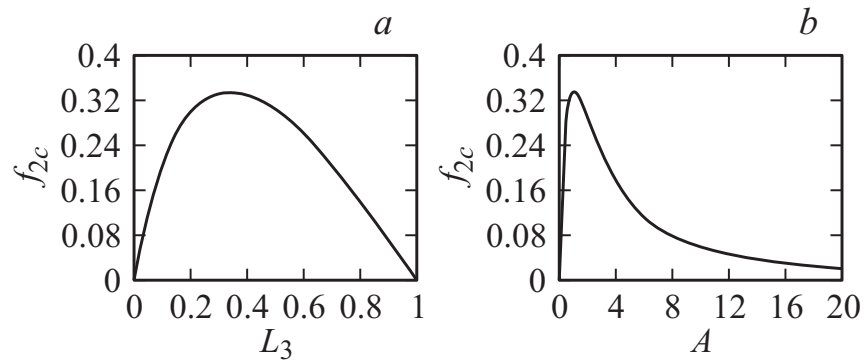

Pис. 3. Зависимость порога протекания от фактора деполяризации $L_{3}(a)$ и от отношения $A(b)$ в модели хаотически ориентированных эллипсоидов.

оба этих случая отвечают предельному случаю частиц с большим аспектным отношением $A^{\prime} \rightarrow \infty$.

Заметим, однако, что более строгий подход показывает, что в случае нанотрубок простая модель вытянутых эллипсоидов пригодна лишь для качественного описания задачи, но дает неправильную асимптотику порога протекания при $A \rightarrow \infty$, требуя определенной модификации [27].

\section{4. Пример: слоистые сферические частицы}

В качестве простого примера частиц с неоднородным распределением внутреннего поля рассмотрим случай одинаковых по структуре сферических рассеивателей, представляющих собой однородное ядро с проницаемостью $\varepsilon_{3}$ в оболочке с проницаемостью $\varepsilon_{2}$ (геометрия рассеивателя показана на рис. 4). Размеры таких рассеивателей могут быть произвольны, но достаточно малы, чтобы не выходить за рамки квазистатического приближения, с сохранением относительных объемов ядра и оболочки.

Решение задачи для одиночного рассеивателя в однородном внешнем поле для этого случая хорошо известно (см., например, [28]), так что $\Lambda(r)$ можно записать в явном виде. Поле внутри такой частицы неоднородно, причем $\Lambda(r)$ представляет собой тензор

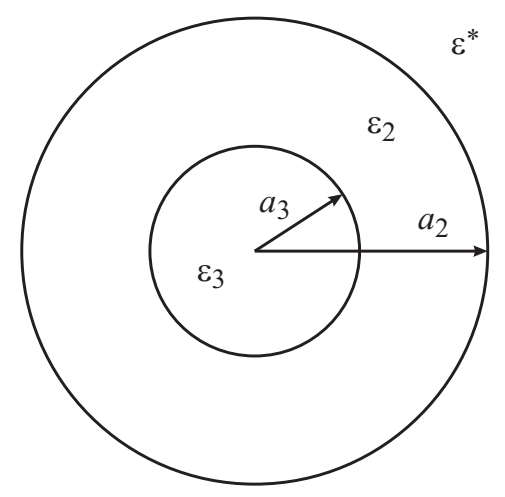

Рис. 4. Структура двуслойной частицы. второго ранга. Однако из-за сферической симметрии рассеивателя результат усреднения по объему $V_{2}$ в (22) сводится к умножению на скаляр, так что достаточно провести усреднение произвольной диагональной компоненты $\Lambda_{z z}(r)$ этого тензора. Таким образом, можно записать

$$
\left\langle\varepsilon\left(r_{2}\right) \Lambda\left(r_{2}\right)\right\rangle_{V_{2}}=\left\langle\varepsilon\left(r_{2}\right) \Lambda_{z z}\left(r_{2}\right)\right\rangle_{V_{2}} .
$$

Подставив это $\Lambda(r)$ в (16) в случае MGA и в (22) в случае ЕМА и проведя усреднение по объему частицы, нетрудно получить явный вид выражения для $\varepsilon^{*}$ в MGA или явный вид уравнения для $\varepsilon^{*}$ в приближении ЕМА (см. Приложение 2). Аналогичные вычисления можно проделать и в общем случае слоистых сферических частиц, для которых известно аналитическое решение задачи о рассеянии на одиночной частице [22,28].

\section{5. Анизотропный случай}

Полученные выше соотношения (16) и (22) coxpaняют свой вид и в случае анизотропных сред с тензорным локальным значением диэлектрической проницаемости $\varepsilon(r)$ (локальная анизотропия), а также для статистически анизотропных сред, когда анизотропия возникает после усреднения из-за наличия преимущественной ориентации частиц (структурная анизотропия). В последнем случае $\varepsilon(r)$ может сводиться к умножению на скаляр из-за отсутствия локальной („микроскопической“) анизотропии, тогда как $\varepsilon^{*}$ становится тензором, что отражает наличие „макроскопической“ анизотропии, т.е. анизотропии в среднем.

Для использования записанных выше соотношений (16), (22) в анизотропном случае достаточно заменить, где это необходимо, скаляры на соответствующие тензоры, деление на скаляр - умножением на соответствующую обращенную матрицу, добавляя символы единичных матриц у скалярных слагаемых и не забывая о некоммутативности тензорных величин при осуществлении алгебраических преобразований.

Так, например, в анизотропном случае приближение Максвелла-Гарнетта (16) переходит в следующее выражение для тензора эффективной проницаемости среды:

$$
\varepsilon^{*}=\left(f_{1} \varepsilon_{1} 1+f_{2}\langle\varepsilon(r) \Lambda(r)\rangle_{v}\right)\left(f_{1} 1+f_{2}\langle\Lambda(r)\rangle_{v}\right)^{-1},
$$

где $\varepsilon^{*}, \varepsilon_{1}, \varepsilon(r)$ и $\Lambda(r)$ - тензоры, 1 - символ единичной матрицы, а усреднение по объему $v$ может дополняться статистическим усреднением по флуктуирующим параметрам эффективной ячейки.

В случае одинаково ориентированных однородных эллипсоидов со скалярной диэлектрической проницаемостью $\varepsilon_{2}$ в изотропной среде с проницаемостью $\varepsilon_{1}$ соотношение (8) для поля внутри эллипсоида сохраняет свой вид, причем $\Lambda(r)(9)$ переходит в тензор

$$
\Lambda=\varepsilon_{1}\left(\varepsilon_{1} 1+L\left(\varepsilon_{2}-\varepsilon_{1}\right)\right)^{-1},
$$

где $L-$ хорошо известный тензор деполяризации эллипсоида [24]. Подставляя это выражение в MGA (26) (где 
символы усреднения в данном случае можно опустить), получаем тензорное выражение, которое распадается на три аналогичных соотношения

$$
\varepsilon_{j}^{*}=\left(f_{1} \varepsilon_{1}+f_{2} \varepsilon_{2} \Lambda_{j}\right)\left(f_{1}+f_{2} \Lambda_{j}\right)^{-1},
$$

связывающие собственные значения $\varepsilon_{j}^{*}$ тензора $\varepsilon^{*}$ с собственными значениями $\Lambda_{j}=\varepsilon_{1}\left(\varepsilon_{2}+L_{j}\left(\varepsilon_{1}-\varepsilon_{2}\right)\right)^{-1}$ тензора $\Lambda$.

Для хаотически ориентированных эллипсоидов $\varepsilon^{*}$ снова становится скаляром, а усреднение по эффективному рассеивателю в (26) означает усреднение по ориентациям частиц. Для тензора $\Lambda$ такое усреднение сводится к вычислению $1 / 3$ следа, что дает

$$
\varepsilon^{*}=\left(f_{1} \varepsilon_{1} 1+f_{2} \varepsilon_{2} \frac{1}{3} \operatorname{Sp} \Lambda\right)\left(f_{1} 1+f_{2} \frac{1}{3} \operatorname{Sp} \Lambda\right)^{-1} .
$$

В случае частично ориентированных эллипсоидов приближение MGA приводит к анизотропному значению $\varepsilon^{*}$, для которого усреднение в (26) сводится к усреднению по ориентациям эллипсоидов [29].

Построение приближения эффективной среды в анизотропном случае оказывается более сложным, поскольку в этом приближении необходимо знать распределение поля внутри рассеивающей частицы (т.е. вид тензора $\left.\Lambda\left(r_{2}\right)\right)$, помещенной в анизотропную среду. В последнем случае при нахождении эффективных параметров в приближении ЕМА в литературе иногда без достаточных оснований используют тензор деполяризации эллипсоида в изотропной среде [30,31].

Даже в относительно простом случае однородного эллипсоида, когда поле $\mathrm{E}$ внутри рассеивателя оказывается однородным (так что тензор $\Lambda\left(r_{2}\right)$ не зависит от $r$ ), выражение (27) для тензора $\Lambda$ требует определенной модификации, поскольку факторы деполяризации эллипсоида в анизотропной среде становятся зависящими не только от геометрии эллипсоида, но и от анизотропии среды [32]. Так, вместо исходного эллипсоида с уравнением поверхности $\left\langle x A^{-2} x\right\rangle=1$, где $A=\operatorname{diag}\left(a_{i}\right)$ - диагональный тензор из полуосей эллипсоида $a_{i}$, при построении факторов деполяризации приходится рассматривать ,приведенный“ эллипсоид с уравнением $\left\langle x \sqrt{\varepsilon^{*}} A^{-2} \sqrt{\varepsilon^{*}} x\right\rangle=1$, где $\varepsilon^{*}-$ тензор диэлектрической проницаемости среды. Поэтому в простейшей структурно-анизотропной модели мононаправленных эллипсоидов с изотропными диэлектрическими проницаемостями, когда направления главных осей $\varepsilon^{*}$ и эллипсоидов совпадают, если принять для материала среды шаровую форму ячейки, то из (20) можно получить следующие уравнения ЕМА:

$$
\begin{gathered}
f_{1} \frac{\varepsilon_{1}-\varepsilon_{j}^{*}}{\varepsilon_{1}+2 \varepsilon_{j}^{*}}+f_{2} \frac{1-L_{j}}{2}\left(\varepsilon_{2}-\varepsilon_{j}^{*}\right) \\
\times\left(\varepsilon_{j}^{*}+L_{j}\left(\varepsilon_{2}-\varepsilon_{j}^{*}\right)\right)^{-1}=0 .
\end{gathered}
$$

Здесь $\varepsilon_{j}^{*}-$ собственные значения тензора эффективной диэлектрической проницаемости среды $\varepsilon^{*}$, а $L_{j}-$ факторы деполяризации „приведенного“ эллипсоида. В случае шаровых частиц $L_{j}=1 / 3$ и (30) переходит в обычное уравнение ЕMA (21). Однако в общем случае уравнения (30) нельзя рассматривать независимо, поскольку факторы деполяризации $L_{j}$ здесь зависят от $\varepsilon_{j}^{*}$, так что (30) представляет собой сложную связанную систему из трех уравнений.

\section{Заключение}

В настоящей работе мы рассмотрели простую схему получения приближений Максвелла-Гарнетта и эффективной среды в квазистатическом приближении для случая среды, содержащей макроскопические рассеиватели произвольной формы, состава и ориентаций. Такой подход позволяет легко получить различные формы рассматриваемых приближений для разных моделей эффективных рассеивателей, включая описание частиц сложной формы, неоднородных частиц, а также общий случай частиц с анизотропным заполнением, помещенных в анизотропную среду. При этом построение всех указанных приближений основано на использовании решения задачи о поляризации выбранной модели „эффективного рассеивателя“, помещенного в однородное внешнее поле.

\section{Приложение 1}

Переход от (1) к (3) содержит операцию деления на вектор, использование которой довольно очевидно, но требует небольшого пояснения. На первый взгляд соотношение (3) не имеет строгого математического смысла, поскольку при заданных векторах $\langle D\rangle$ и $\langle E\rangle$ уравнение (1) не определяет однозначно величины $\varepsilon^{*}$, которая в общем случае является тензором. В действительности даже в случае тензорного $\varepsilon^{*}$ здесь мы имеем не недоопределенную, а разрешимую переопределенную систему, поскольку направление $\langle E\rangle$ может быть произвольным. Использование традиционных обозначений позволяет легко доказать допустимость в данном случае „сокращения на вектор“, но теряет в наглядности.

\section{Приложение 2}

Для частиц, представляющих собой шар в оболочке (рис. 4), решение задачи о поляризации в однородном внешнем поле хорошо известно (см., например, [22]). При этом поле в ядре частицы оказывается однородным, а поле в оболочке слагается из однородной компоненты и поля диполя, которое не дает вклада в усредненную по объему величину (22). Опуская эту дипольную компоненту, можно записать

$$
\begin{aligned}
\Lambda(r)= & \frac{3 \varepsilon^{*}}{\left(\varepsilon_{2}+2 \varepsilon^{*}\right)\left(\varepsilon_{3}+2 \varepsilon_{2}\right)+2\left(\varepsilon_{2}-\varepsilon^{*}\right)\left(\varepsilon_{3}-\varepsilon_{2}\right) \beta} \\
& \times \begin{cases}3 \varepsilon_{2}, & r<a_{2}, \\
\left(\varepsilon_{3}+2 \varepsilon_{2}\right), & a_{2}<r<a_{1},\end{cases}
\end{aligned}
$$


где $\beta=\left(a_{3} / a_{2}\right)^{3}$. Подстановка этого выражения в качестве $\Lambda_{2}$ в (19) приводит к следующему уравнению для $\varepsilon^{*}$ :

$$
\begin{aligned}
& 0=f_{1} \frac{\varepsilon_{1}-\varepsilon^{*}}{\varepsilon_{1}+2 \varepsilon^{*}} \\
& +f_{2}\left(\frac{3 \varepsilon^{*}\left(\varepsilon_{3}+2 \varepsilon_{2}\right)+\beta\left(\varepsilon_{2}-\varepsilon_{3}\right)}{\left(\varepsilon_{2}+2 \varepsilon^{*}\right)\left(\varepsilon_{3}+2 \varepsilon_{2}\right)+2\left(\varepsilon_{2}-\varepsilon^{*}\right)\left(\varepsilon_{3}-\varepsilon_{2}\right) \beta}-1\right) .
\end{aligned}
$$

Структура этого уравнения вполне аналогична более простой модели (21) для однородных сфер, и оно также приводит к квадратному уравнению для $\varepsilon^{*}$, однако оно содержит два дополнительных свободных параметра $\varepsilon_{3}$ и $\beta$, что обеспечивает большую гибкость этого приближения.

\section{Список литературы}

[1] Thouless D.J. // Phys. Rep. 1974. Vol. 13. P. 94-142.

[2] Landauer R. // AIP Conference Proceedings. 1978. Vol. 40. P. 2-45.

[3] Bruggeman D.A.G. // Ann. Phys. 1935. Vol. 23. P. 636-664.

[4] Bommeli F., Degiorgi L., Forro L., de Heer W.A. // The Science and Technology of Carbon Nanotubes. Ch. 9. Ed. by K. Tanaka, T. Yamabe, K. Fukui. Elsevier, 1999.

[5] Lu W., Dong J. // Phys. Rev. B 2000. Vol. 63. P. 033401.

[6] Kim H., Abdata A.A., Macosko C.W. // Macromolecules. 2010. Vol. 43. P. 6515-6530.

[7] Cai W., Shalaev V. Optical Metamaterials. Fundamentals and Applications. Springer, 2010.

[8] Виноградов П., Дорофеенко А.В., Зухди С. // УФН. 2008. T. 178 . C. $511-518$.

[9] Sheng P. // Phys. Rev. Lett. 1980. Vol. 45. P. 60-63.

[10] Mc Lachlan D.S. // Sol. Stat. Commun. 1989. Vol. 72. P. $831-834$

[11] Brosseau C. // J. Appl. Phys. 2002. Vol. 91. P. 3197-3204.

[12] Maxwell Garnett J.C. // Phil. Trans. R. Soc. London, 1904. Vol. A203. P. $385-420$.

[13] Marton J.P., Lemon J.R. // Phys. Rev. B. 1971. Vol. 4. P. $271-280$

[14] Petrov Yu.I. Physics of Small Particles. M.: Nauka, 1982 (in Russian).

[15] Milton G.V. The Theory of Composites. Cambridge Univ. Press, 2004. 719 p.

[16] Hashin Z., Shtrikman S. // J. Mech. Phys. Solids. 1962. Vol. 10. P. 343-352.

[17] Aspnes D.E. // Thin Solid Ftlms. 1982. Vol. 89. P. 249-262.

[18] Shin F.G., Yeung Y.Y., Tsui W.L. // J. Mater. Sci. Lett. 1990. Vol. 9. P. 1002-1004.

[19] Iglesias T.P., Fernandez J.P. // J. Mater. Sci. Lett. 2001. Vol. 20. P. 1333-1334.

[20] Stroud D. // Phys. Rev. B. 1975. Vol. 12. P. 3368-3373.

[21] Bergman D.J., Stroud D. // Sol. Stat. Phys. 1992. Vol. 46. P. $148-269$.

[22] Sihvola A. Electromagnetic Mixing Formulas and Applications, Electromagnetic Wave Series 47. London: IEE Publishing, 1999.

[23] Huang J.P., Yu K.W. // Phys. Rep. 2006. Vol. 431. P. 87-172.

[24] Рытов С.М., Кравцов Ю.А., Татарский В.И. Введение в статистическую радиофизику. Т. 1,2. М.: Наука, 1978.
[25] Yaghjian A.D. // Proc. IEEE. 1980. Vol. 68. P. 248-283.

[26] Landau L.D., Lifshitz E.M. Electrodynamics of Continuous Media. Oxford: Pergamon Press, 1984.

[27] Lagarkov A.N., Sarychev A.K. // Phys. Rev. B. 1996. Vol. 53. P. 6318-6336.

[28] Sihvola A., Lindell I.V. // J. El. Wav. Appl. 1988. Vol. 2. P. 741-756.

[29] Sihvola A., Kong J.A. // IEEE Trans. Geopsci. Rem. Sens. 1988. Vol. 26. P. 420-429.

[30] Smith G.B. // Opt. Commun. 1989. Vol. 71. P. 279.

[31] Grangvist C.G. et al. // Renew. Energ. 1996. Vol. 8. P. 530.

[32] Апресян Л.А., Власов Д.В. // ЖТФ. 2014. Т. 84. С. 23-28. 